\title{
Patterns of Potentially Inappropriate Bladder Antimuscarinic Use in People with Dementia: A Retrospective Cohort Study
}

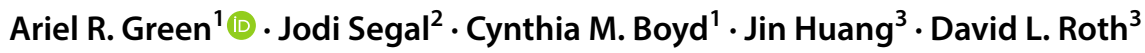

Published online: 12 February 2020

(c) The Author(s) 2020

\begin{abstract}
Background Emergency department (ED) visits or hospitalizations should prompt review of the patient's medications after discharge and targeted deprescribing to reduce ongoing risks.

Objective To see if this is happening, we sought to examine the proportion of serious falls and delirium that were followed by a prescription fill for a bladder antimuscarinic. Our secondary objective was to identify concurrent use of other anticholinergics, cholinesterase inhibitors (ChEIs), and diuretics among people with dementia taking bladder antimuscarinics.

Methods Retrospective descriptive study using data from the National Health and Aging Trends Study (2011-2014) linked with Medicare claims from 2010-2014. The primary outcome was the proportion of serious falls or delirium that were followed by a bladder antimuscarinic prescription fill within 12 months. The secondary outcome was the proportion of individuals concurrently receiving prescriptions for bladder antimuscarinics, other strong anticholinergics, ChEIs, and diuretics. Results During the observation period, $8.88 \%$ of people with dementia filled a bladder antimuscarinic prescription. Over one-third (35\%) initiated use after being identified as having dementia. Many used bladder antimuscarinics concurrently with other strong anticholinergics (19\%), diuretics (42\%), and ChEIs (32\%). The majority (76\%) of serious falls or delirium among people with dementia and bladder antimuscarinic use were followed by a bladder antimuscarinic prescription within 12 months.

Conclusions In this descriptive study, bladder antimuscarinic prescriptions were often filled irrespective of dementia status, serious falls, or delirium. Concurrent use of other anticholinergics, diuretics, and ChEIs was common. Falls and delirium should trigger review of the medication list after discharge and targeted deprescribing in people with dementia.
\end{abstract}

\section{Introduction}

Urinary incontinence is common [1] and highly burdensome for older adults with dementia; it is associated with falls and fractures (e.g., by causing frail individuals to rise multiple times at night to urinate), decreased quality of life for patients and caregivers, hospitalizations, nursing home admissions, and death [2-6]. Behavioral strategies (e.g.,

Ariel R. Green

ariel@jhmi.edu

1 Division of Geriatric Medicine and Gerontology, Department of Medicine, Johns Hopkins University School of Medicine, Mason F. Lord Building, Center Tower, 5200 Eastern Ave., 7th Floor, Baltimore, MD 21224, USA

2 Division of General Internal Medicine, Department of Medicine, Johns Hopkins University School of Medicine, Baltimore, MD, USA

3 Center on Aging and Health, Johns Hopkins University, Baltimore, MD, USA scheduled voiding) are the recommended first-line therapies for urge incontinence [7], but are challenging to implement in people with dementia [8]. Bladder antimuscarinics are commonly prescribed [9]. However, clinical practice guidelines and expert panels urge caution in using these agents in people with impaired cognition or frailty, for whom the benefits may be attenuated and the harms magnified [7, 10, 11].

Bladder antimuscarinic drugs are strong antagonists of muscarinic receptors and thus have the potential to cause a range of side effects, including dry mouth, blurred vision, impaired cognitive performance, constipation, falls, and delirium [12-14]. These side effects-particularly the latter two-often lead to emergency department (ED) visits and hospitalizations, and may threaten the ability of people with dementia to remain living at home instead of institutional settings, a key patient- and family-centered outcome. In addition, higher total anticholinergic burden from the accumulated effects of multiple medicines with anticholinergic properties is associated with poorer cognitive performance and greater mortality among older adults $[15,16]$. 


\section{Key Points}

Emergency department (ED) visits or hospitalizations should prompt review of the patient's medications after discharge and targeted deprescribing to reduce ongoing risks.

This study found that most serious falls and delirium among community-dwelling older adults with dementia and bladder antimuscarinic use were followed by bladder antimuscarinic prescription fills within 12 months after the event.

Among patients with dementia who used bladder antimuscarinics, concurrent use of other anticholinergics, diuretics, and cholinesterase inhibitors was also common.

In addition to the concern about anticholinergic side effects, bladder antimuscarinics may be involved in adverse drug-drug interactions [17]. For example, because bladder antimuscarinics work by blocking the activity of acetylcholine at muscarinic receptors, they may directly counteract the already-modest therapeutic effect of cholinesterase inhibitors (ChEIs), one of two US Food and Drug Administration (FDA)-approved pharmacotherapies for dementia [18]. Concurrent treatment with bladder antimuscarinics and ChEIs may lead to faster rates of functional decline as compared with ChEIs alone [19]. Bladder antimuscarinics may also be involved in prescribing cascades, in which the effect of one drug is misinterpreted as a new medical condition, triggering an additional prescription to treat it. For example, the prescription of a bladder antimuscarinic to manage incontinence in a patient who is taking a diuretic may represent one such cascade [20]. Prescribing cascades are major contributors to unnecessary and harmful polypharmacy in older adults [20].

Ideally, patients with dementia who have ED visits or hospitalizations due to falls or delirium would have a careful review of all of their medications after discharge, a discussion with patients and caregivers would ensue about the relative benefits and risks of each drug, and select medications would be stopped or continued appropriately. To begin to understand whether these best practices are occurring, we evaluated the proportion of ED visits and hospitalizations due to serious falls and delirium that were followed by a prescription fill for a bladder antimuscarinic in a cohort of community-dwelling older adults with dementia. Our secondary, related, objective was to identify concurrent use of other anticholinergics, ChEIs, and diuretics among older adults with dementia taking bladder antimuscarinics.

\section{Methods}

\subsection{Design, Data Sources, and Sample Population}

This was a retrospective cohort study leveraging 4 years of data from the National Health and Aging Trends Study (NHATS) (2011-2014) that had been linked with Medicare Part A, B, and D claims from 2010 to 2014. Medicare Part A includes information from inpatient hospitalizations, Part B includes information from hospital outpatient and physician visits, and Part D includes information from the Medicare prescription drug benefit, including claims for drug dispensings in outpatient settings. NHATS is an ongoing, nationally representative, prospective cohort study of Medicare enrollees in the USA aged $\geq 65$ years. Participants are selected from all age groups, with oversampling of the oldest ( $\geq 90$ years) and of non-Hispanic Black persons. Inperson interviews are conducted annually in participants' homes and include questionnaires and physical and cognitive assessments. Persons familiar with the participant's daily routines act as proxy respondents if the older adult is unable to respond. The baseline wave of NHATS (2011) included 8245 individuals. We included those who were communitydwelling $(n=7197)$.

We identified a cohort of participants with possible or probable dementia from 1 May 2011 through 31 October 2014. Follow-up began on the date of dementia classification and continued through death or 31 December 2014, whichever came first. A validated algorithm using three types of information identifies participants in NHATS who have probable dementia [21]: (1) Self or proxy report of being told by a doctor that the sample person has dementia; (2) a score $\leq 1.5$ standard deviations below mean in at least two domains; [21] or (3) a score $\geq 2$ out of 8 on the AD8 Dementia Screening Interview [22, 23] administered to proxies. A cut-point of $\leq 1.5$ standard deviations below the mean in one domain was used for possible dementia. The NHATS possible or probable dementia classification has been shown to have specificity of $84 \%$ against persons classified as having normal cognition [21]. To capture varying degrees of cognitive impairment, we included individuals in the cohort if they had been classified as having probable dementia at one NHATS assessment or possible dementia at two consecutive NHATS assessments. The date of entry into our cohort was the date of the NHATS assessment at which the participant was classified as having probable dementia or the second consecutive date at which the participant was classified as having possible dementia. For inclusion in the cohort, individuals were also required to have 6 months of continuous enrollment in Parts A, B, and D of traditional fee-for-service Medicare prior to the date of cohort entry 
and 1 month of continuous enrollment after the month of cohort entry (Fig. 1).

\subsection{Serious Falls and Delirium}

We identified serious falls in two ways. First, the presence of ED and inpatient claims with a fall-related E code (8800-8889) or an injury code for nonpathological skull, facial, cervical, clavicle, humeral, forearm, pelvic, hip, fibula, tibia, or ankle fractures $(80,000-80,619,8070-8072$, $8080-8089,81,000-81,419,8180-8251$, or 8270-8291), brain injury $(85,200-85,239)$, or dislocation of the hip, knee, shoulder, or jaw $(8300-83,219,83,500-83,513$, or $83,630-83,660$ ) was considered a serious fall [24]. In the absence of a fall-related $\mathrm{E}$ code, the event was considered a fall-related injury, and therefore a serious fall, if there was an emergency department or inpatient claim for any of these injuries and there was no motor vehicle accident E code (8100-8199). We also identified ED and inpatient claims with delirium $(290.11,290.12,290.13,290.20,290.3$, 290.41, 290.42, 290.43, 290.8, 290.9, 293.0-293.9, 348.30, $348.31,348.39,349.82,780.0,780.09,780.02,780.97)$ [25-27].

\subsection{Antimuscarinic Use}

We searched the Part D claims from 6 months prior to cohort entry to 31 December 2014 for prescription fills of at least a 30-day supply for the following bladder antimuscarinic drugs: oxybutynin, tolterodine, fesoterodine, solifenacin, darifenacin, trospium, and flavoxate (Fig. 2). Part D claims include the name, strength, route of administration, date dispensed, quantity dispensed, and days' supply for each drug. The end date of use was considered the date on which the last prescription's supply should have ended. A medication supply gap of $\geq 30$ days was used to define treatment discontinuation. Discrete episodes of bladder antimuscarinic use were defined as a prescription fill of any bladder antimuscarinic agent following a discontinuation period of $\geq 30$ days [28]. Date of death was ascertained from the Medicare Master Beneficiary Summary file.

\subsection{Exposure to Other Anticholinergic Medicines, Diuretics, and Cholinesterase Inhibitors}

We calculated the average daily Anticholinergic Cognitive Burden scale (ACB) score of participants from cohort entry
Fig. 1 Overview of cohort selection

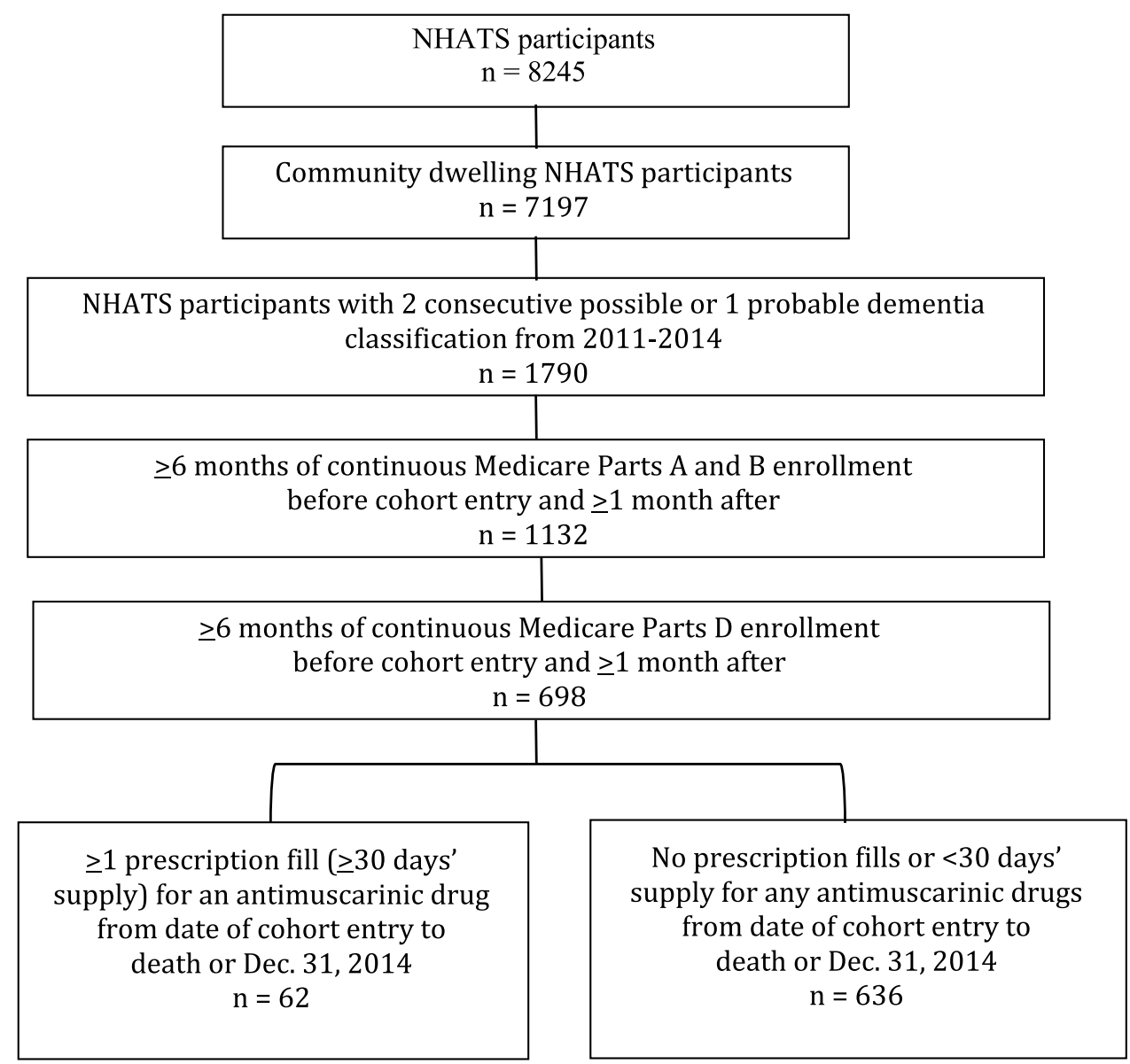




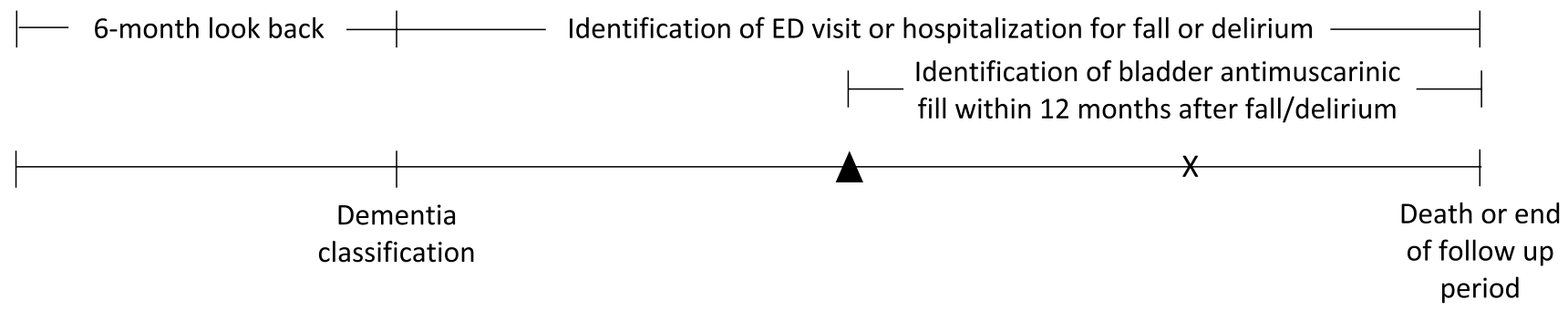

$\Delta=$ Emergency Department visit or hospitalization for fall or delirium

$X=$ Prescription fill for bladder antimuscarinic after Emergency Department visit or hospitalization for fall or delirium

Fig. 2 Study design

through death or 31 December 2014, whichever came first. The ACB is an expert-derived drug-rating scale to quantify anticholinergic burden $[15,29]$. Each medicine is rated on an ordinal scale from 1 to $3(1=$ possible anticholinergic cognitive effects; 2 and $3=$ definite anticholinergic cognitive effects). The total score is the sum of ACB scores of all medications a patient is taking. The average daily ACB score incorporates the number of drugs, the duration of use of each drug, and the anticholinergic rating (i.e., ACB Level 1,2 , or 3 ) of each drug. We included all prescription fills for anticholinergic drugs in calculating ACB scores. We also calculated the proportion of participants who filled prescriptions for non-bladder antimuscarinic medicines with ACB scores of 2 or 3. Lastly, we examined the proportion of bladder antimuscarinic users concurrently receiving (1) the ChEIs tacrine, galantamine, donepezil, or rivastigmine, and (2) the diuretics chlorthalidone, indapamide, hydrochlorothiazide, furosemide, bumetanide, torsemide, triamterene, amiloride, spironolactone, or eplerenone. Concurrent use was defined as days' supplies that overlapped for at least 7 days.

\subsection{Analytic Methods}

We used the Chi-square statistic to compare proportions and simple linear regression to compare means between bladder antimuscarinic users and non-users. We then calculated various measures related to bladder antimuscarinic use: total days of bladder antimuscarinic use, number of discrete episodes of bladder antimuscarinic use, and initiation of bladder antimuscarinic use after dementia classification in NHATS. We used standard descriptive methods to examine the proportion of individuals concurrently receiving prescriptions for bladder antimuscarinics, other strongly anticholinergic medications, ChEIs, and diuretics. Finally, we calculated the primary outcome-the proportion of serious falls or delirium that were followed by a bladder antimuscarinic prescription fill within 12 months after the ED visit or hospitalization. Analyses were performed using SAS version 9.3 (SAS Institute, Inc., Cary, NC, USA).

\section{Results}

There were 1790 community-dwelling NHATS participants with probable or possible dementia classifications from 1 May 2011 through 31 October 2014. After applying the Medicare-related inclusion criteria, the study cohort consisted of 698 people (Fig. 1). The mean (SD) age was 83.4 (7.77) years, $462(66.2 \%)$ were women, and the majority (372; 53.3\%) were White, non-Hispanic. During the followup period, $62(8.88 \%)$ people with dementia filled a bladder antimuscarinic prescription of at least a 30-day supply. Among bladder antimuscarinic users, 22 (35.5\%) had no bladder antimuscarinic prescription fills in the 6-month look-back period and initiated use after dementia classification. Table 1 provides characteristics of the cohort members, stratified by exposure to bladder antimuscarinics. Compared to nonusers, individuals prescribed bladder antimuscarinics tended to be younger and were more likely to take five or more medications; they also tended to have better cognitive scores, higher co-morbidity scores, and a greater number of functional impairments. Oxybutynin and solifenacin were the most commonly prescribed bladder antimuscarinics (51.6\% and $27.4 \%$, respectively). Bladder antimuscarinic users had an average of 357.8 (329.1) total days' supply and 1.77 (1.23) discrete episodes of bladder antimuscarinic use.

The average total daily ACB score was 2.03 (1.68) among people taking bladder antimuscarinics (not including the bladder antimuscarinic) and 1.62 (1.24) among people who did not take bladder antimuscarinics. Among bladder antimuscarinic users, $19.4 \%$ concurrently took other medications with ACB scores of 2 or 3 (Table 2). The most common anticholinergic drugs taken concurrently with bladder antimuscarinics were cyclobenzaprine, meclizine, 
Table 1 Characteristics of the cohort ${ }^{\mathrm{a}}$

\begin{tabular}{|c|c|c|c|c|}
\hline Variable & Overall $(n=698)$ & $\begin{array}{l}\text { Bladder antimus- } \\
\text { carinic use }(n=62)^{\mathrm{b}}\end{array}$ & $\begin{array}{l}\text { No bladder antimus- } \\
\text { carinic use }(n=636)\end{array}$ & $p$ value \\
\hline $\operatorname{Age}(y)$, mean $(S E)^{c}$ & $83.4(7.8)$ & $82.7(7.7)$ & $83.5(7.8)$ & 0.46 \\
\hline Female, $n(\%)$ & $462(66.2)$ & $44(71.0)$ & $418(65.7)$ & 0.40 \\
\hline Race, ${ }^{\mathrm{d}} n(\%)$ & & & & 0.54 \\
\hline White, non-Hispanic & $372(53.3)$ & $34(54.8)$ & $338(53.1)$ & \\
\hline Black, non-Hispanic & $206(29.5)$ & $15(24.2)$ & $191(30.0)$ & \\
\hline Hispanic or other & $120(17.2)$ & $13(21.0)$ & $107(16.8)$ & \\
\hline Marital status, $n(\%)$ & & & & 0.04 \\
\hline Married or living with partner & $211(30.5)$ & $26(41.9)$ & $185(29.3)$ & \\
\hline Other & $482(69.6)$ & $36(58.1)$ & $446(70.7)$ & \\
\hline Living alone, $n(\%)$ & $214(31.0)$ & $17(27.4)$ & $197(31.3)$ & 0.53 \\
\hline No. of ADL and IADL disabilities, ${ }^{\mathrm{e}}$ mean (SE) & $5.39(3.9)$ & $6.47(3.7)$ & $5.28(3.9)$ & 0.02 \\
\hline Charlson co-morbidity index, mean (SE) & $2.36(2.5)$ & $2.82(2.4)$ & $2.31(2.5)$ & 0.12 \\
\hline Average (SE) daily $\mathrm{ACB}^{\mathrm{f}}$ score & $1.66(1.3)$ & $2.03(1.7)$ & $1.62(1.2)$ & 0.06 \\
\hline Total cognitive score, ${ }^{\mathrm{g}}$ mean (SE) & $7.87(4.7)$ & $8.63(3.9)$ & $7.79(4.8)$ & 0.23 \\
\hline$\geq 5$ medications in 6 months prior to cohort entry, $n(\%)$ & $566(84.7)$ & $56(90.3)$ & $510(84.2)$ & 0.20 \\
\hline Follow-up period (months), median (IQR) & $29.01(25.7)$ & $30.5(22.0)$ & $29.0(26.5)$ & 0.13 \\
\hline
\end{tabular}

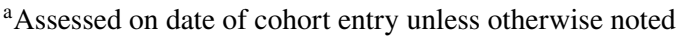

${ }^{b}$ Bladder antimuscarinic prescription fill of $\geq 30$ days' supply at any point during follow-up

${ }^{\mathrm{c}} \mathrm{SE}$ : Standard error

${ }^{\mathrm{d}}$ Reference: White

${ }^{\mathrm{e}} \mathrm{ADL}$ /IADLs: ADLs (activities of daily living) include bathing, eating, dressing, toileting, getting out of bed, getting around inside the home, and leaving home. IADLs (instrumental activities of daily living) include cooking, managing finances, managing medications, grocery shopping, and doing laundry

${ }^{\mathrm{f}}$ ACB: Anticholinergic Cognitive Burden scale; calculated from cohort entry through death or 31 December 2014, whichever came first; does not include bladder antimuscarinic

${ }^{\mathrm{g}}$ Cognitive testing assessed memory (immediate and delayed 10-word recall), orientation (date, month, year, day of week; naming president and vice president), and executive function (clock drawing test); higher scores are better

paroxetine, and quetiapine. In addition, $41.9 \%$ of bladder antimuscarinic users concurrently received a diuretic and $32.3 \%$ received a ChEI.

From the date of dementia classification through death or 31 December 2014 [median 30.5 (interquartile range (IQR) 22.0) months for bladder antimuscarinic users and 29.0 (IQR $26.5)$ months for non-users, $p=0.13], 180(25.8 \%)$ participants had an ED visit or hospitalization due to a fall and 116
(16.6\%) for delirium. Among individuals who filled bladder antimuscarinic prescriptions, 24 participants (38.7\%) had at least one serious fall and $17(27.4 \%)$ had at least one episode of delirium. There were 56 total ED visits or hospitalizations due to a serious fall or delirium; 32 of $56(57.1 \%)$ falls or delirium resulted in hospitalization and the average length of stay was 8.0 (10.3) days. Because 15 of the 56 ED visits or hospitalizations occurred less than 12 months before the
Table 2 Patterns of use of bladder antimuscarinics, other anticholinergics, cholinesterase inhibitors, and diuretics

\begin{tabular}{lc}
\hline Variable & No. (\%) \\
\hline Concurrent use $^{\mathrm{a}}$ of other strongly anticholinergic medications & $12(19.4)$ \\
Concurrent use of cholinesterase inhibitors $^{20(32.3)}$ \\
Concurrent use of diuretics & $26(41.9)$ \\
Bladder antimuscarinic prescription fill within 12 months after Emergency Department visit or & $31(75.6)$ \\
hospitalization for fall or delirium $^{\mathrm{b}}$ & \\
\hline${ }^{\mathrm{a}}$ Concurrent use: Days' supplies that overlapped for at least 7 days & \\
${ }^{\mathrm{b}}$ Among 62 patients with antimuscarinic use, there were 56 emergency department visits or hospitaliza- \\
tions due to a fall or delirium; 15 of these events occurred less than 12 months before the patient's death or \\
31 December 2014 and we were unable to determine whether there was an antimuscarinic prescription fill \\
within 12 months after the fall or delirium. Percentage is based on remaining 41 events
\end{tabular}


patient's death or 31 December 2014, we were unable to determine whether there was a bladder antimuscarinic prescription fill within 12 months after the event. Among the remaining 41 events, 31 (75.6\%) were followed by a bladder antimuscarinic prescription fill within 12 months after the ED visit or hospitalization (Table 2).

\section{Discussion}

To begin to understand whether best practices are occurring with respect to deprescribing of potentially inappropriate medications in patients with dementia, this study examined the proportion of ED visits and hospitalizations due to serious falls and delirium that were followed by a prescription fill for a bladder antimuscarinic in a cohort of community-dwelling older adults with dementia. A second, related aim was to identify concurrent use of other anticholinergics, ChEIs, and diuretics among patients with dementia taking bladder antimuscarinics. We found that the majority of serious falls and delirium among people with dementia and bladder antimuscarinic use-76\%-were followed by bladder antimuscarinic prescription fills within 12 months after the event. We also found evidence of prescribing practices that could lead to adverse drug-drug interactions: $19 \%$ of people with dementia who used bladder antimuscarinics concurrently received other strongly anticholinergic medicines, $42 \%$ concurrently received diuretics, and 32\% received ChEIs.

These findings echo other recent studies that examined PIM use in older adults with dementia, though few studies have examined whether use persists in the outpatient setting after trigger events such as ED visits or hospitalizations. One study found that anticholinergic prevalence among older adults with dementia was 23\% [30]. Another found that 31\% of Medicare Advantage Prescription Drug Plan members who were newly initiated on a bladder antimuscarinic had a drug-drug or drug-disease interaction; dementia was the most common such interaction, present in $11 \%$ of people initiated on a bladder antimuscarinic drug [17]. Previous research has shown that multiple anticholinergic drugs are often prescribed concurrently, causing anticholinergic side effects to accumulate [31, 32], and that bladder antimuscarinic use is common in individuals receiving ChEIs for treatment of dementia. A previous study that examined pharmacy claims for Iowa's Medicaid population found that 35\% of those receiving ChEIs were receiving at least one other anticholinergic medicine, with bladder antimuscarinics among the most common [18]. The use of a bladder antimuscarinic in this setting may represent a prescribing cascade, as ChEIs may theoretically cause or exacerbate urinary incontinence [33]. Dual use of ChEIs and antimuscarinics may not only render both medicines less effective, but may lead to accelerated functional decline compared to use of ChEIs alone [19].

We are not aware of other studies that have examined the rate of concurrent use of bladder antimuscarinics and diuretics. This practice is an illustrative example of therapeutic competition, a common and burdensome scenario for older adults with multiple chronic conditions, in which treatment of one condition exacerbates the second, and subsequent treatment of the second condition aggravates the first [34]. Between 35 and $50 \%$ of heart failure patients suffer from urinary incontinence. This may be due, at least in part, to the use of diuretics to treat heart failure and their effects of increasing urinary frequency and urgency [35]. Bladder antimuscarinics may then be prescribed, resulting in a prescribing cascade. Bladder antimuscarinic agents may also theoretically worsen heart failure, though this putative association requires further study [36]. There are numerous strategies for addressing incontinence in patients with heart failure; prescription of bladder antimuscarinics is not the preferred management approach due to the high risk of adverse effects with little likelihood of benefit [35].

An ED visit or hospitalization should serve as a prompt for outpatient clinicians to review patient medications with an eye toward deprescribing after discharge, yet there are many barriers to optimizing patient drug regimens, including after such trigger events. Clinicians in the outpatient setting may not know that patients have been hospitalized or may lack complete hospital records at post-discharge visits [37]. Thus, they may not recognize that the patient's ED visit or hospitalization could have been due to an anticholinergicinduced adverse event. In addition, clinicians are often hesitant to discontinue medications that are being prescribed by other providers involved in the patient's care [38], or they may have incomplete information about patient drug regimens, particularly after patients transition from the hospital to the outpatient setting. For example, a previous study that found that $96 \%$ of primary-care provider medication lists contained discrepancies compared with what the patient was actually taking [39]. This problem may be magnified for patients with dementia, who are often unable to give clear medication histories and may not attend visits with caregivers who are knowledgeable about their drug regimens [38]. Clinicians may also assume that serious falls and delirium are unavoidable consequences of dementia and may not connect these events to medications.

Clinicians may continue to prescribe potentially inappropriate medications, such as bladder antimuscarinics, because behavioral interventions are perceived as too time-consuming to explain or burdensome for caregivers, or because 
clinicians lack support to implement them [40]. Nonpharmacologic interventions such as caregiver training and comanagement with non-physician care managers improve a number of patient and caregiver outcomes, but these have not been widely disseminated [41-44]. Medicare provides a limited home health benefit, but does not cover personal care services for people with dementia or other nonpharmacological interventions such as respite care, environmental assessments and modifications, and caregiver training [45]. Each of these may reduce use of potentially inappropriate medications. Interventions such as multidisciplinary team medication review and academic detailing have been the most effective at reducing polypharmacy and use of PIMs, but these are resource-intensive and challenging to adapt to a variety of clinical settings [46]. Patient-directed educational interventions have also been shown to decrease use of other classes of potentially inappropriate medication, such as benzodiazepines [47], and should be expanded to target inappropriate antimuscarinic prescriptions in people with dementia and to involve caregivers. Computerized decision support tools have shown promise for triggering medication reviews and reducing inappropriate prescribing in older adults; such tools could be especially useful for identifying patients who have experienced recent serious falls or delirium, but there remain substantial barriers to their widespread dissemination [48]. Moreover, none of the existing computerized decision support interventions have focused on patients with dementia - an area that warrants further research.

Our study has limitations. Misclassification of dementia is possible because our definition of dementia is based on the limited set of cognitive tests or proxy assessments used in NHATS, rather than a full neurocognitive evaluation. Misclassification of antimuscarinic use is also possible using prescription drug claims. For example, prescriptions may be filled but never taken [49]. In addition, delirium is likely to be undercoded in claims data [50]. We identified $119(18.1 \%)$ ED visits or hospitalizations due to delirium in our cohort. This is in line with rates of delirium reported in the literature, which range from 18 to $35 \%$ on hospital admission and 8 to $17 \%$ on presentation to the emergency department [51]. Bladder antimuscarinics may vary in their propensity to cross the blood-brain barrier and affect cognition [52]; we did not assess whether bladder antimuscarinic users with falls or delirium were subsequently switched to a bladder antimuscarinic with a potentially lower risk of adverse cognitive effects. Lastly, prescribing and deprescribing depend on the individual patient context and on discussions that take place in the examination room between patient, caregiver, and clinician. Claims data do not provide information that allows us to know this; it is possible that bladder antimuscarinics were in some cases continued after a thoughtful process of patient-centered communication and decision-making.

\subsection{Conclusions}

Among community-dwelling older adults with dementia, bladder antimuscarinic prescriptions were often filled irrespective of dementia status, serious falls, or delirium. Concurrent use of other anticholinergics, diuretics, and ChEIs was also common. Interventions are needed to help clinicians review medication lists after serious falls and delirium, and deprescribe potentially inappropriate medications, including bladder antimuscarinics, in patients with dementia.

\section{Compliance with Ethical Standards}

Funding Research reported in this publication was conducted with support from the National Institute on Aging under award numbers K23AG054742 (Green) and K24AG056578 (Boyd).

Conflict of Interest Dr. Boyd writes a chapter on multimorbidity for UpToDate, for which she receives a royalty. The other authors have no conflicts of interest that are directly relevant to the content of this article.

Ethics Approval The Johns Hopkins University (JHU) Bloomberg School of Public Health Institutional Review Board oversees NHATS; this study was approved by the JHU School of Medicine IRB.

Open Access This article is licensed under a Creative Commons Attribution-NonCommercial 4.0 International License, which permits any non-commercial use, sharing, adaptation, distribution and reproduction in any medium or format, as long as you give appropriate credit to the original author(s) and the source, provide a link to the Creative Commons licence, and indicate if changes were made. The images or other third party material in this article are included in the article's Creative Commons licence, unless indicated otherwise in a credit line to the material. If material is not included in the article's Creative Commons licence and your intended use is not permitted by statutory regulation or exceeds the permitted use, you will need to obtain permission directly from the copyright holder.To view a copy of this licence, visit http://creativecommons.org/licenses/by-nc/4.0/.

\section{References}

1. Sakakibara R, Uchiyama T, Yamanishi T, Kishi M. Dementia and lower urinary dysfunction: with a reference to anticholinergic use in elderly population. Int J Urol. 2008;15(9):778-88. https://doi. org/10.1111/j.1442-2042.2008.02109.x.

2. Luo X, Chuang CC, Yang E, Zou KH, Araiza AL, Bhagnani T. Prevalence, management and outcomes of medically complex vulnerable elderly patients with urinary incontinence in the United States. Int J Clin Pract. 2015. https://doi.org/10.1111/ijcp.12740.

3. Sexton CC, Coyne KS, Thompson C, Bavendam T, Chen CI, Markland A. Prevalence and effect on health-related quality of life of overactive bladder in older americans: results from the epidemiology of lower urinary tract symptoms study. J Am Geriatr Soc. 2011;59(8):1465-70. https://doi.org/10.111 1/j.1532-5415.2011.03492.x.

4. Brown JS, Vittinghoff E, Wyman JF, Stone KL, Nevitt MC, Ensrud KE, et al. Urinary incontinence: does it increase risk for 
falls and fractures? Study of Osteoporotic Fractures Research Group. J Am Geriatr Soc. 2000;48(7):721-5.

5. Ko Y, Lin SJ, Salmon JW, Bron MS. The impact of urinary incontinence on quality of life of the elderly. Am J Manag Care. 2005;11(4 Suppl):S103-11.

6. Thom DH, Haan MN, Van Den Eeden SK. Medically recognized urinary incontinence and risks of hospitalization, nursing home admission and mortality. Age Ageing. 1997;26(5):367-74.

7. Gormley EA, Lightner DJ, Faraday M, Vasavada SP. Diagnosis and treatment of overactive bladder (non-neurogenic) in adults: AUA/SUFU guideline amendment. J Urol. 2015;193(5):1572-80. https://doi.org/10.1016/j.juro.2015.01.087.

8. Drennan VM, Greenwood N, Cole L, Fader M, Grant R, Rait $\mathrm{G}$, et al. Conservative interventions for incontinence in people with dementia or cognitive impairment, living at home: a systematic review. BMC Geriatr. 2012;12:77. https://doi. org/10.1186/1471-2318-12-77.

9. Kachru N, Carnahan RM, Johnson ML, Aparasu RR. Potentially inappropriate anticholinergic medication use in older adults with dementia. J Am Pharm Assoc (2003). 2015;55(6):603-12. https ://doi.org/10.1331/japha.2015.14288.

10. American Geriatrics Society 2015 updated beers criteria for potentially inappropriate medication use in older adults. J Am Geriatr Soc. 2015;63(11):2227-46. https://doi.org/10.1111/jgs.13702.

11. Lightner DJ, Gomelsky A, Souter L, Vasavada SP. Diagnosis and treatment of overactive bladder (non-neurogenic) in adults: AUA/SUFU guideline amendment 2019. J Urol. 2019:101097ju0000000000000309. https://doi.org/10.1097/ ju.0000000000000309.

12. Mintzer J, Burns A. Anticholinergic side-effects of drugs in elderly people. J R Soc Med. 2000;93(9):457-62.

13. Richardson K, Bennett K, Maidment ID, Fox C, Smithard D, Kenny RA. Use of medications with anticholinergic activity and self-reported injurious falls in older community-dwelling adults. J Am Geriatr Soc. 2015;63(8):1561-9. https://doi.org/10.1111/ jgs. 13543.

14. Green AR, Reifler LM, Bayliss EA, Weffald LA, Boyd CM. Drugs contributing to anticholinergic burden and risk of fall or fallrelated injury among older adults with mild cognitive impairment, dementia and multiple chronic conditions: a retrospective cohort study. Drugs Aging. 2019;36(3):289-97. https://doi.org/10.1007/ s40266-018-00630-z.

15. Campbell N, Boustani M, Limbil T, Ott C, Fox C, Maidment I, et al. The cognitive impact of anticholinergics: a clinical review. Clin Interv Aging. 2009;4:225-33.

16. Fox C, Richardson K, Maidment ID, Savva GM, Matthews FE, Smithard D, et al. Anticholinergic medication use and cognitive impairment in the older population: the medical research council cognitive function and ageing study. J Am Geriatr Soc. 2011;59(8):1477-83. https://doi.org/10.111 1/j.1532-5415.2011.03491.x.

17. Suehs BT, Davis C, Franks B, Yuran TE, Ng D, Bradt J, et al. Effect of potentially inappropriate use of antimuscarinic medications on healthcare use and cost in individuals with overactive bladder. J Am Geriatr Soc. 2016;64(4):779-87. https://doi. org/10.1111/jgs. 14030

18. Carnahan RM, Lund BC, Perry PJ, Chrischilles EA. The concurrent use of anticholinergics and cholinesterase inhibitors: rare event or common practice? J Am Geriatr Soc. 2004;52(12):2082 7. https://doi.org/10.1111/j.1532-5415.2004.52563.x.

19. Sink KM, Thomas J 3rd, Xu H, Craig B, Kritchevsky S, Sands LP. Dual use of bladder anticholinergics and cholinesterase inhibitors: long-term functional and cognitive outcomes. J Am Geriatr Soc. 2008;56(5):847-53. https://doi.org/10.111 1/j.1532-5415.2008.01681.x.
20. Rochon PA, Gurwitz JH. The prescribing cascade revisited. The Lancet. 2017;389(10081):1778-80. https://doi.org/10.1016/S0140 -6736(17)31188-1.

21. Kasper JD, Freedman VA, Spillman BC. Classification of persons by dementia status in the National Health and Aging Trends Study [Internet] Baltimore (MD): Johns Hopkins Bloomberg School of Public Health; 2013. Jul, [cited 2019 Dec 13]. (Technical Paper No. 5). http://www.nhats.org/scripts/documents/NHATS_Demen tia_Technical_Paper_5_Jul2013.pdf.

22. Galvin JE, Roe CM, Powlishta KK, Coats MA, Muich SJ, Grant $\mathrm{E}$, et al. The AD8: a brief informant interview to detect dementia. Neurology. 2005;65(4):559-64. https://doi.org/10.1212/01. wnl.0000172958.95282.2a.

23. Galvin JE, Roe CM, Xiong C, Morris JC. Validity and reliability of the AD8 informant interview in dementia. Neurology. 2006;67(11):19428. https://doi.org/10.1212/01.wnl.0000247042.15547.eb.

24. Tinetti ME, Han L, Lee DSH, McAvay GJ, Peduzzi P, Gross CP, et al. Antihypertensive medications and serious fall injuries in a nationally representative sample of older adults. JAMA Intern Med. 2014;174(4):588-95.

25. Inouye SK, Leo-Summers L, Zhang Y, Bogardus ST Jr, Leslie DL, Agostini JV. A chart-based method for identification of delirium: validation compared with interviewer ratings using the confusion assessment method. J Am Geriatr Soc. 2005;53(2):312-8. https ://doi.org/10.1111/j.1532-5415.2005.53120.x.

26. Katznelson R, Djaiani G, Tait G, Wasowicz M, Sutherland AM, Styra R, et al. Hospital administrative database underestimates delirium rate after cardiac surgery. Can J Anaesth. 2010;57(10):898-902. https://doi.org/10.1007/s1263 0-010-9355-8.

27. Bui LN, Pham VP, Shirkey BA, Swan JT. Effect of delirium motoric subtypes on administrative documentation of delirium in the surgical intensive care unit. J Clin Monit Comput. 2017;31(3):631-40. https://doi.org/10.1007/s10877-016-9873-1.

28. Sussman D, Yehoshua A, Kowalski J, Lee W, Kish J, Chaudhari $S$ et al. Adherence and persistence of mirabegron and anticholinergic therapies in patients with overactive bladder: a realworld claims data analysis. Int J Clin Pract. 2017. https://doi. org/10.1111/ijcp.12824.

29. Campbell NL, Maidment I, Fox C, Khan B, Boustani M. The 2012 update to the anticholinergic cognitive burden scale. J Am Geriatr Soc. 2013;61(S1):S142-3. https://doi.org/10.1111/jgs.2013.61. issue-s1.

30. Sura SD, Carnahan RM, Chen H, Aparasu RR. Prevalence and determinants of anticholinergic medication use in elderly dementia patients. Drugs Aging. 2013;30(10):837-44. https://doi. org/10.1007/s40266-013-0104-x.

31. Parkinson L, Magin PJ, Thomson A, Byles JE, Caughey GE, Etherton-Beer C, et al. Anticholinergic burden in older women: not seeing the wood for the trees? Med J Aust. 2015;202(2):91-4.

32. Green AR, Reifler LM, Boyd CM, Weffald LA, Bayliss EA. Medication profiles of patients with cognitive impairment and high anticholinergic burden. Drugs Aging. 2018;35(3):223-32. https ://doi.org/10.1007/s40266-018-0522-x.

33. Gill SS, Mamdani M, Naglie G, Streiner DL, Bronskill SE, Kopp A, et al. A prescribing cascade involving cholinesterase inhibitors and anticholinergic drugs. Arch Intern Med. 2005;165(7):808-13. https://doi.org/10.1001/archinte.165.7.808.

34. Mallet L, Spinewine A, Huang A. The challenge of managing drug interactions in elderly people. Lancet. 2007;370(9582):185-91. https://doi.org/10.1016/s0140-6736(07)61092-7.

35. Tannenbaum C, Johnell K. Managing therapeutic competition in patients with heart failure, lower urinary tract symptoms and incontinence. Drugs Aging. 2014;31(2):93-101. https://doi. org/10.1007/s40266-013-0145-1. 
36. Andersson KE, Sarawate C, Kahler KH, Stanley EL, Kulkarni AS. Cardiovascular morbidity, heart rates and use of antimuscarinics in patients with overactive bladder. BJU Int. 2010;106(2):268-74. https://doi.org/10.1111/j.1464-410X.2009.09073.x.

37. Kripalani S, LeFevre F, Phillips CO, Williams MV, Basaviah P, Baker DW. Deficits in communication and information transfer between hospital-based and primary care physicians: implications for patient safety and continuity of care. JAMA. 2007;297(8):83141. https://doi.org/10.1001/jama.297.8.831.

38. Green AR, Lee P, Reeve E, Wolff JL, Chen CCG, Kruzan R, et al. Clinicians' perspectives on barriers and enablers of optimal prescribing in patients with dementia and coexisting conditions. J Am Board Fam Med. 2019;32(3):383-91. https://doi.org/10.3122/ jabfm.2019.03.180335.

39. Frank C, Godwin M, Verma S, Kelly A, Birenbaum A, Seguin R, et al. What drugs are our frail elderly patients taking? Do drugs they take or fail to take put them at increased risk of interactions and inappropriate medication use? Can Fam Physician. 2001;47:1198-204.

40. Kerns JW, Winter JD, Winter KM, Boyd T, Etz RS. Primary care physician perspectives about antipsychotics and other medications for symptoms of dementia. J Am Board Fam Med. 2018;31(1):921. https://doi.org/10.3122/jabfm.2018.01.170230.

41. Gitlin LN, Kales HC, Lyketsos CG. Nonpharmacologic management of behavioral symptoms in dementia. JAMA. 2012;308(19):2020-9. https://doi.org/10.1001/jama.2012.36918.

42. Callahan CM, Boustani MA, Unverzagt FW, Austrom MG, Damush TM, Perkins AJ, et al. Effectiveness of collaborative care for older adults with Alzheimer disease in primary care: a randomized controlled trial. JAMA. 2006;295(18):2148-57. https ://doi.org/10.1001/jama.295.18.2148.

43. Jennings LA, Tan Z, Wenger NS, Cook EA, Han W, McCreath HE, et al. Quality of care provided by a comprehensive dementia care comanagement program. J Am Geriatr Soc. 2016;64(8):1724-30. https://doi.org/10.1111/jgs.14251.

44. Bass DM, Judge KS, Snow AL, Wilson NL, Morgan R, Looman WJ, et al. Caregiver outcomes of partners in dementia care: effect of a care coordination program for veterans with dementia and their family members and friends. J Am Geriatr Soc. 2013;61(8):1377-86. https://doi.org/10.1111/jgs.12362.
45. Cabin WD. Moving toward Medicare home health coverage for persons with Alzheimer's disease. J Gerontol Soc Work. 2008;51(1-2):77-86. https://doi.org/10.1080/016343708019675 62.

46. Gnjidic D, Le Couteur DG, Kouladjian L, Hilmer SN. Deprescribing trials: methods to reduce polypharmacy and the impact on prescribing and clinical outcomes. Clin Geriatr Med. 2012;28(2):237-53. https://doi.org/10.1016/j.cger.2012.01.006.

47. Tannenbaum C, Martin P, Tamblyn R, Benedetti A, Ahmed S. Reduction of inappropriate benzodiazepine prescriptions among older adults through direct patient education: the EMPOWER cluster randomized trial. JAMA Intern Med. 2014;174(6):890-8. https://doi.org/10.1001/jamainternmed.2014.949.

48. Clyne B, Bradley MC, Hughes C, Fahey T, Lapane KL. Electronic prescribing and other forms of technology to reduce inappropriate medication use and polypharmacy in older people: a review of current evidence. Clin Geriatr Med. 2012;28(2):301-22. https:// doi.org/10.1016/j.cger.2012.01.009.

49. Funk MJ, Landi SN. Misclassification in administrative claims data: quantifying the impact on treatment effect estimates. Curr Epidemiol Rep. 2014;1(4):175-85. https://doi.org/10.1007/s4047 1-014-0027-z.

50. Israni J, Lesser A, Kent T, Ko K. Delirium as a predictor of mortality in US Medicare beneficiaries discharged from the emergency department: a national claims-level analysis up to 12 months. BMJ Open. 2018;8(5):e021258. https://doi.org/10.1136/ bmjopen-2017-021258.

51. Inouye SK, Westendorp RG, Saczynski JS. Delirium in elderly people. Lancet. 2014;383(9920):911-22. https://doi.org/10.1016/ s0140-6736(13)60688-1.

52. Scheife R, Takeda M. Central nervous system safety of anticholinergic drugs for the treatment of overactive bladder in the elderly. Clin Ther. 2005;27(2):144-53. https://doi.org/10.1016/j.clint hera.2005.02.014 